Hydroécol. Appl. (1994) Tome 6 Vol 1-2, pp. 175-196

\title{
Etude du peuplement zooplanctonique de la retenue de Pareloup (Aveyron, France)
}

\section{Study of the zooplankton population in Pareloup reservoir (Aveyron, France)}

\section{P. Francisco, J. Rey}

Laboratoire d'Hydrobiologie (CNRS, URA 695), Université Paul Sabatier, 118 route de Narbonne, F-31062 Toulouse Cedex, France.

Résumé. - Le peuplement zooplanctonique de la retenue de Pareloup suivi de 1983 à 1987 est essentiellement représenté par Diaptomus cyaneus cyaneus, Cyclops vicinus vicinus, Acanthocyclops robustus, Daphnia longispina et Bosmina longirostris. La communauté est dominée par les Copépodes : diaptomides en hiver et au printemps, Cyclopides en été et en automne. Les Cladocères sont caractérisés par le développement de Daphnia longispina au début de l'été, celui de Bosmina longirostris en période automnale. La contribution des Rotifères, maximale en hiver et au printemps, ne dépasse généralement pas $10 \%$. En période estivale, la densité moyenne maximale des Crustacés (34 ind./litre) est relevée en 1984.

L'estimation de l'impact du broutage par les Crustacés sur la communauté phytoplanctonique révèle le rôle prépondérant des herbivores et des petits brouteurs (Diaptomidae, Bosminidae) à la fin de l'hiver-début du printemps, et des petits brouteurs (Bosminidae, nauplii de Cyclopides) à la fin de l'automne. En été, les grands brouteurs constituent les principaux régulateurs des populations algales $(44,5 \%$ du volume potentiel d'eau filtrée).

La biomasse moyenne estivale de zooplancton montre une valeur maximale de $75 \mathrm{mg} / \mathrm{m}^{3}$ en 1983. Durant cette période, la biomasse moyenne varie d'une année à l'autre d'environ un facteur 2 pour les Cladocères, un facteur 4 pour les Copépodes. Selon les années, on observe une prédominance variable des groupes de Crustacés (Copépodes en 1983, Cladocères en 1987) ou des espèces de Cladocères (Daphnia en 1986, Bosmina en 1987). Cette variabilité interannuelle de la densité et de la structure du peuplement zooplanctonique est expliqué par le contrôle exercé par les poissons zooplanctophages peuplant la retenue.

Mots-clés. - Zooplancton, structure du peuplement, évolution saisonnière, densité, retenue. 
Summary. - The zooplankton population of Pareloup reservoir, studied from 1983 to 1987 , is essentially composed of Diaptomus cyaneus cyaneus, Cyclops vicinus vicinus, Acanthocyclops robustus, Daphnia longispina and Bosmina longirostris. The community is dominated by Copepods: Diaptomus in winter and spring and Cyclopods in summer and autumn. The Cladocerans are characterized by the development of Daphnia Iongispina in early summer and that of Bosmina longirostris in the autumn. Rotifers, at their maximum in winter and spring, do not generally exceed $10 \%$. In summer, the maximum average density of Crustaceans (34 ind./lit) is found in 1984.

Estimation of the impact of grazing on the phytoplankton community by Crustaceans reveals the preponderant role of herbivores and small grazers (Diaptomidae, Bosminidae) at the end of winter-early spring, and of small grazers (Bosminidae, nauplii of Cyclopidae) at the end of autumn. In summer, large grazers represent the prime regulators of algal populations $(44.5 \%$ of the potential volume of filtered water).

The average summer zooplankton biomass reaches a maximum value of $75 \mathrm{mg} / \mathrm{m}^{3}$ in 1983. During this period, the average biomass varies from one year to another by a factor of about 2 for the Cladocerans and 4 for the Copepods. Depending on the year, one notes a variable predominance of groups of Crustaceans (Copepods in 1983, Cladocerans in 1987) or Cladoceran species (Daphnia in 1986. Bosmina in 1987). This year-to-year variability in the density and structure of the zooplankton population can be explained by the regulatory control exercised by zooplankton-grazing fish in the reservoir.

Key-words. - Zooplankton, population structure, seasonal evolution, density, reservoir.

\section{INTRODUCTION}

L'impact des facteurs physico-chimiques et biologiques (nutrition, prédation) sur le développement des populations zooplanctoniques varie en fonction de la saison et du niveau trophique du lac (Mc Queen et al., 1986).

L'étude des relations prédateursproies régissant l'évolution des zooplanctontes revêt un intérêt particulier pour la compréhension de la dynamique fonctionnelle des écosystèmes lacustres.

La construction d'un modèle fonctionnel en vue de la gestion d'un lac (Thébault et Salençon, 1993) nécessite une approche de la communauté zooplanctonique orientée vers des in- teractions existantes entre les niveaux de la pyramide trophique.

L'étude du peuplement zooplanctonique du lac de Pareloup présentée dans ce travail a donc été conduite parallèlement à d'autres travaux (Capblancq et al., 1988; Lavandier, 1990; Labroue, 1990; Le Jolivet, 1988).

\section{SITE, MATÉRIEL ET MÉTHODES}

Le lac de Pareloup est situé au sudouest du Massif Central, à une altitude de 800 mètres. Avec une superficie de $12,6 \mathrm{~km}^{2}$ et un volume d'eau de 169 millions de mètres cubes, il constitue l'une des plus vastes retenues de France. Le régime ther- 
mique (fig. 1) fait apparaître une stratification de la masse d'eau, stable de juin à septembre. Les températures moyennes des deux strates 0-10 mètres et 10-25 mètres sont alors respectivement de 18,6 et $13,3^{\circ} \mathrm{C}$. Durant la période d'isothermie, la moyenne sur la colonne d'eau est de $7,6^{\circ} \mathrm{C}$. En été, la zone euphotique correspond généralement à l'épilimnion; sa profondeur moyenne est de 8,25 mètres. Sur le plan trophique, le lac de Pareloup est de type mésotrophe.

L'échantillonnage a été réalisé au cours de deux périodes d'études:

— du 7 juillet 1983 au 11 juin 1985 ,

— du 25 juin 1986 au 18 décembre 1987 .

L'absence de prélèvement au mois de janvier et février est due aux conditions difficiles d'accès au site durant l'hiver.

Les relevés sont effectués en un point représentatif du peuplement planctonique situé dans la partie

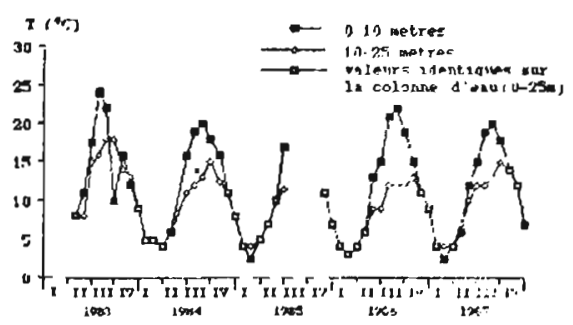

Fig. 1. - Evolution de la température (valeurs moyennes) du lac de Pareloup au cours de la période étudiée.

Fig. 1. - Evolution of temperature (mean values) in Pareloup lake during the period under study. ouest du lac (plateforme E.D.F.) selon deux méthodes complémentaires:

- l'une, quantitative, est basée sur l'utilisation d'une pompe immergée balayant toute la colonne d'eau par tranches successives d'une amplitude de 5 mètres. Un prélèvement de 5 litres d'eau est filtré sur un tamis de vide de maille $20 \mu \mathrm{m}$ pour chaque tranche d'eau prospectée;

- l'autre, qualitative, consiste en une capture d'organismes au moyen d'un filet à plancton remonté du fond jusqu'à la surface.

Après sédimentation, les organismes ont été dénombrés et mesurés à l'aide d'un microscope inversé pour les petites formes (Rotiferes, stades naupliens) et d'une loupe binoculaire $(G: 12,5 \times 4)$ pour les formes de plus grande taille (Cladocères et adultes de Copépodes).

Les densités, exprimées en nombre d'individus par litre, sont calculées pour toute la colonne d'eau par la moyenne pondérée de l'effectif de chaque tranche d'eau.

Les valeurs de biomasse ont été calculées d'après les relations taillepoids empruntées à la littérature (Dumont et al., 1975; Bottrel et al., 1976).

Les relations phytoplancton-zooplancton sont envisagées par le biais d'une classification fonctionnelle du zooplancton (Sprules, 1984), basée sur la taille des organismes et leur écologie trophique:

- petits brouteurs (nauplii des Copépodes et tous les Cladocères de taille inférieure à $0,5 \mathrm{~mm}$ ), 
- grands brouteurs (Cladocères d'une taille supérieure à $0,5 \mathrm{~mm}$ ),

- herbivores (copépodites et adultes de Diaptomides),

- carnivores (copépodites et adultes de Cyclopides). II faut toutefois noter que cette catégorie regroupe plutôt des organismes omnivores que des carnivores stricts.

L'impact du broutage par les zooplanctontes est estimé à partir des taux de filtration moyens établis par Baucheff-Nielsen (1988), pour chaque groupe de filtreurs:

- 1,29 ml/ind./jour pour les petits brouteurs,

- 2,34 $\mathrm{ml} /$ ind./jour pour les grands brouteurs, vores.

- 2,03 ml/ind./jour pour les herbi-

\section{RÉSULTATS}

\subsection{Composition du peuplement zooplanctonique}

Le lac de Pareloup est colonisé par 16 espèces de Crustacés et 10 espèces de Rotifères:

\section{Cladocères}

Daphniidae :

- Daphnia longispina (O.F. MÜLLER, 1785)

- Ceriodaphnia quadrangula (O.F. MÜLLER, 1785)

Bosminidae :

- Bosmina longirostris (O.F. MÜLLER, 1785)
Chydoridae :

- Chydorus sphaericus (O.F. MÜLLER, 1785)

- Alona affinis (LEYDIG, 1860)

- Alona costata (SARS, 1862)

- Alonella nana (BAIRD, 1843)

- Leydigia quadrangularis (LEYDIG, 1860)

Holopedidae :

- Holopedium gibberum (ZAD$\mathrm{DACH}, 1855)$

Sididae :

- Sida crystallina (O.F. MÜLLER, 1776)

- Diaphanosoma brachyurum (LIEVIN, 1848)

Macrothricidae :

- llyocryptus sordidus (LIEVIN, 1848)

\section{Copépodes}

Calanidae :

- Diaptomus cyaneus cyaneus (GURNEY, 1909)

Cyclopidae:

- Cyclops vicinus vicinus (ULIANINE, 1875)

- Acanthocyclops robustus (G.O. SARS, 1863)

- Macrocyclops albidus (JURINE, 1820)

- Thermocyclops crassus (FISCHER, 1853)

\section{Rotifères :}

- Kellicottia longispina (KELLICOTT, 1879)

- Keratella cochlearis (GOSSE, 1951)

- Filinia longiseta (EHRB, 1834)

- Asplanchna priodonta (GOSSE, 1950) 
- Ploesoma hudsoni (HERRICK, 1885)

- Polyarthra vulgaris (CARLIN, 1943)

- Conochilus unicornis (ROUSSE-

LET, 1892)

- Synchaeta sp.

- Ascomorpha sp.

- Hexarthra sp.

Certaines des espèces rencontrées, particulièrement chez les Cladocères Chydoridae, sont tychoplanctoniques. Parmi les formes eupélagiques, seules les espèces caractéristiques présentes dans la majorité des prélèvements sont prises en compte dans l'analyse du peuplement.

Ce sont: les deux Cladocères Daphnia longispina, Bosmina longirostris, le Calanide Diaptomus cyaneus cyaneus. Acanthocyclops robustus et Cyclops vicinus vicinus sont regroupés sous le terme de Cyclopides. Les Rotifères sont considérés globalement.

Ceriodaphnia quadrangula, Cladocère présent pendant une courte période estivale, est pris en compte dans les groupes fonctionnels "petits brouteurs" ou "grands brouteurs" selon les classes de taille enregistrées.

\section{2 Évolution saisonnière de la densité et de la biomasse totales des groupes zooplanctoniques}

\subsubsection{Densité}

Le suivi des populations de Crustacés (fig. 2a, 2b) en 1983, 1984, 1986 et 1987, montre un schéma évolutif si- milaire avec deux pics d'abondance maximale, l'un au printemps-début été, l'autre en automne. Ces deux phases de développement sont séparées par une période de déclin estival.

Chez les Cladocères (fig. 2a), le premier pic est plus ou moins précoce selon les années (fin juin en 1984, 86 , 87; mi-juillet en 1983). Le pic automnal présente un développement comparable à celui du pic estival en 1983 et 1986; il est nettement moins marqué en 1984 et 1987.

Les densités maximales sont voisines en 1983 (20 ind.// à la mi-juillet), 1984 (21,5 ind.// le 21 juin) et 1986 (18,6 ind./l le 30 juin). La plus forte valeur de densité est enregistrée au printemps 1985 (33,3 ind.// le 11 juin). Les valeurs les plus basses sont observées en 1986, année où le maximum se situe à 13,2 ind./litre le 30 juin.
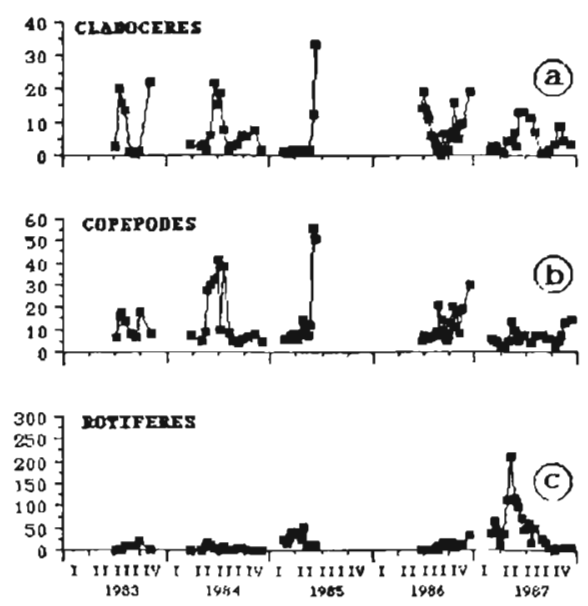

Fig. 2. - Evolution de la densité (ind./litre) des trois groupes du zooplancton de 1983 à 1987. Fig. 2. - Evolution of the density (ind./l) of the three zooplankton groups from 1983 to 1987. 
Les Copépodes (fig. 2b) montrent deux pics d'abondance, estival et automnal, en 1983,86 et 87 , années où les valeurs de densités ne dépassent pas 20 ind./litre. II faut en excepter toutefois décembre 1987, période où le développement automnal des Copépodes atteint 29,8 ind./litre le 18 décembre (soit $61,8 \%$ des Crustacés), ce qui constitue l'effectif maximum observé pour cette saison au cours des cinq années d'étude.

En 1984, le peuplement estival des Copépodes montre, comparativement aux autres années, des valeurs de densités presque deux fois supérieures $(41,1$ et 37,7 ind./I les 2 et 24 juillet respectivement). Le développement automnal est par contre peu important : 7,8 ind./litre. Comme pour les Cladocères, la densité maximale est enregistrée au printemps 1985 (55,8 ind./l le 3 juin).

Comparativement, peu représentés en 1983, 1984 et 1986, les Rotiferes (fig. 2c) dominent le peuplement zooplanctonique de mars à mai 1985 avec des densités comprises entre 18 et 51 ind./litre. En 1987, avec une moyenne de 49,6 ind./litre pour les mois échantillonnés, ils dominent le peuplement de mars à septembre. Le pic d'abondance observé le 13 mai (212 ind./I.) constitue l'effectif maximum de toute la période d'étude.

\subsubsection{Biomasse}

Les valeurs maximales de la biomasse correspondent pour les Cladocères (fig. 3a) et les Rotifères (fig. 3c) aux maxima de densité observés. En revanche, pour les Copépodes (fig. 3b), en raison d'un fort écart pondéral entre les différents stades de développement, on observe parfois une discordance entre les deux paramètres.

Les plus fortes valeurs de biomasse sont enregistrées au printemps 1985: 63 et $92,5 \mu \mathrm{g}$ P.S./litre le 11 juin pour les Cladocères et les Copépodes respectivement. Elles correspondent à l'augmentation des effectifs déjà observée (fig. 2a, 2b). Cependant, ces observations révèlent la domination des Copépodes tout au long de la période vernale, situation qui n'apparait pas en termes de densité. En outre, à la différence des valeurs de densité, la biomasse printanière copépodienne présente deux maxima: le premier (24 avril) coincide avec une phase à faibles effectifs
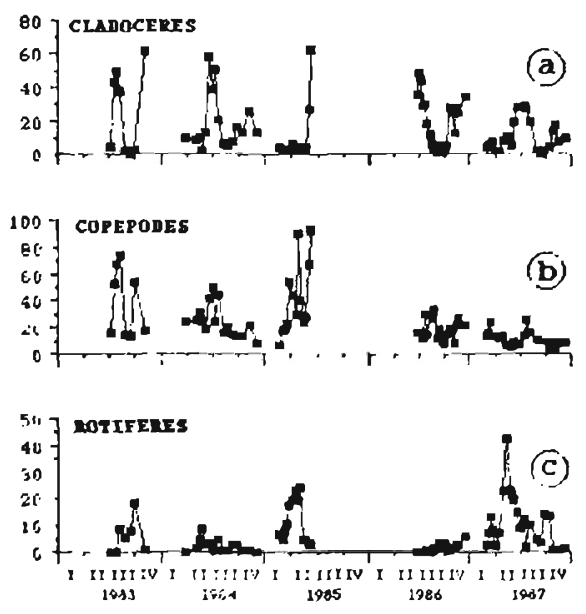

Fig. 3. - Evolution de la biomasse ( $\mu$ g P.S./I) des trois groupes du zooplancton de 1983 a 1987.

Fig. 3. - Evolution of the biomass ( $\mu \mathrm{g}$ D.W.II) of the three zooplankton groups from 1983 to 1987. 
mais constituée à près de $75 \%$ de formes adultes et subadultes à fort poids moyen individuel, le second ( 3 juin) correspond à une augmentation du nombre des individus de Cyclopides majoritairement représentés par de jeunes stades (fig. 6a, 6b).

En 1983, 84, 86 et 87, l'évolution de la biomasse montre deux pics saisonniers (été, automne) avec cependant des variations interannuelles dues, soit à une contribution saisonnière plus ou moins grande de l'un ou l'autre groupe soit à des écarts plus ou moins marqués entre les deux phases de développement. Ainsi, en 1983, les Copépodes sont majoritaires en été et forment $54,7 \%$ de la biomasse estivale, mais sont largement dominés en automne par les Cladocères qui représentent $77 \%$ de la biomasse le 9 novembre 1983. En 1984, la biomasse estivale présente des valeurs deux fois supérieures aux valeurs automnales, pour les deux groupes de Crustacés.

Comme cela a déjà été noté précédemment, les discordances observées entre valeurs de biomasse et densités correspondantes sont liées au cycle vital des Copépodes. Les biomasses relativement élevées enregistrées début avril et juillet 1987, en période de faibles effectifs (fig. $2 b$, $3 b)$, sont dues à la forte contribution de stades adultes et subadultes présents durant cette période comme le montre l'analyse ultérieure de la structure du peuplement (fig. 8b).

Inversement, la chute des valeurs de biomasse en automne 1986 et 1987, malgré une forte augmentation du nombre des individus, résulte de la prédominance, à cette époque, des stades naupliens et copépodites à faible poids individuel.

Les Rotifères (fig. 3c) contribuent plus ou moins fortement à la biomasse zooplanctonique. Leur contribution est la plus forte en période hivernale et printanière $(24,5 \mu \mathrm{g}$ P.S./I le 2 mai 1985). La forte valeur enregistrée le 12 mai 1987 (42,4 ug P.S./, soit environ $60 \%$ de la biomasse totale) résulte peut-être d'une surestimation de l'effectif moyen relatif à un biais de l'échantillonnage.

\subsection{Evolution de la structure du peuplement}

La structure du peuplement zooplanctonique a été étudiée pour les deux espèces dominantes de Cladocères, Daphnia longispina et Bosmina longirostris, pour le Diaptomide Diaptomus cyaneus cyaneus et pour les Cyclopides.

En 1983 (fig. 4a, 4b), le peuplement estival est constitué de mi-juillet à mi-août, de Copépodes et de Cladocères en proportions à peu près égales, avec prédominance de Daphnia pour les Cladocères et de Diaptomus pour les Copépodes. De mi-août à fin septembre, les Copépodes, largement dominés par le Diaptomide, constituent plus de $80 \%$ du peuplement.

Les plus fortes valeurs de densités estivales sont atteintes en juillet: 14 ind./ litre le 17/07 pour Daphnia, 
Ind./1

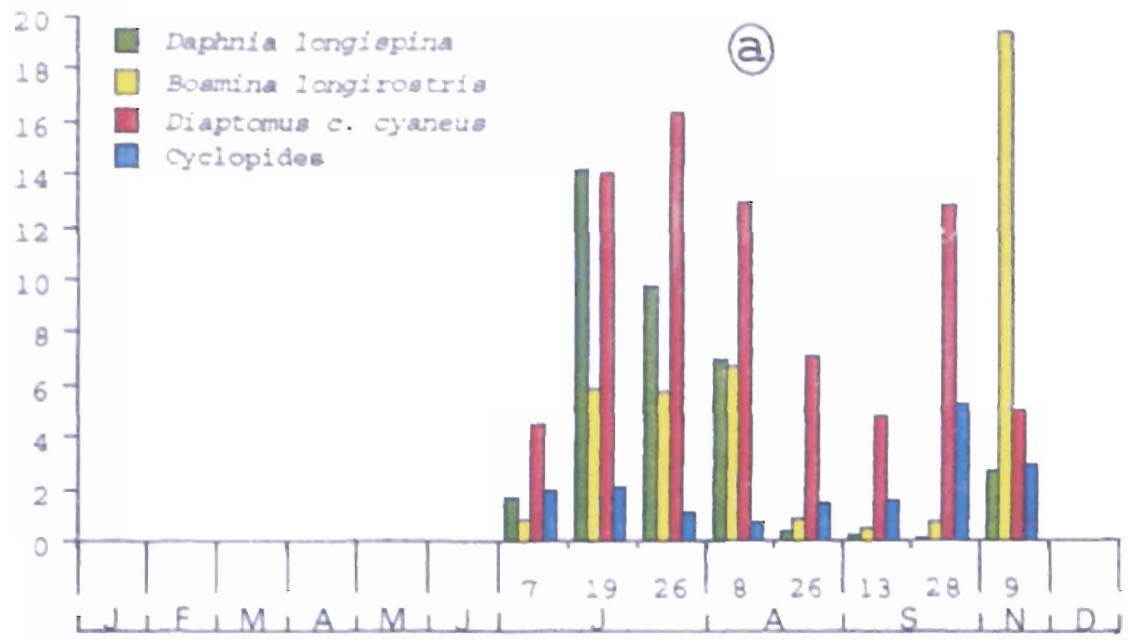

Densite ers of ?

(b)

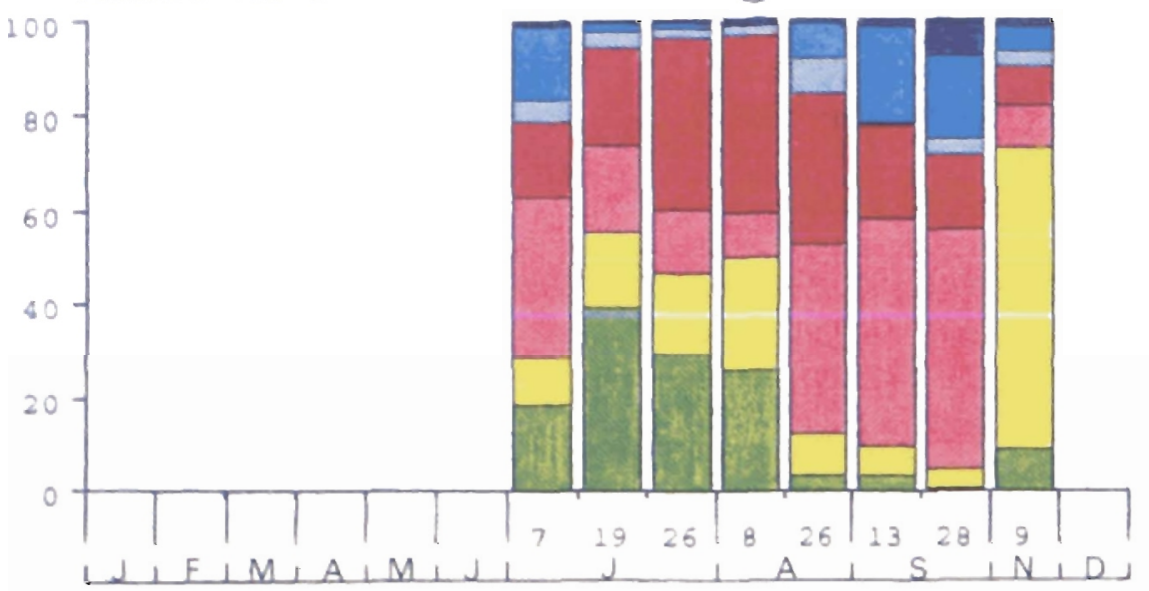

Q Daphnia longispina $\square$ D. c. cyaneus: Napli1 $\square$ Oycl. I Naupliti

$\square$ Bomina longirostris D. C. cyaneus: Ad.+ Cop. D Cycl. : Cop.

a cyel. : Aa.

Fig. 4. - Structure du peuplement zooplanctonique en 1983; a : densité, b: composition relative.

Fig. 4. - Structure of the zooplankton population in 1983; a: density, b: relative abundance. 
16,5 ind./ litre le 26/07 pour Diaptomus.

Le peuplement automnal se caractérise par le développement de Bosmina longirostris qui, avec 20 ind./l le 9 novembre, représente à elle seule $64 \%$ des Crustacés.

Excepté fin septembre, les effectifs de Cyclopides sont toujours inférieurs à 2 ind./litre.

En 1984 (fig. 5a, 5b), les Copépodes se révèlent largement majoritaires.

Au printemps et en été, ils représentent en moyenne $75 \%$ des Crustacés. En automne, ils s'équilibrent avec les Cladocères. Le peuplement copépodien printanier est dominé par Diaptomus puis atteint une abondance maximale le 24 mai (25 ind./l dont $96 \%$ de nauplii). Le peuplement estival est caractérisé par le développement des Cyclopidae dont les pics de juillet ( 40 et 37 ind.// les 2 et 24/07 respectivement) correspondent également à une forte production de stades naupliens.

Comparativement aux autres années, Daphnia longispina est, en 1984, relativement peu représentée. La prédation exercée sur les Daphniidae par les stades adultes et subadultes de Cyclopides, bien représentés en 1984, contribue probablement au déclin estival du Cladocère. Santer (1993) montre l'existence au printemps d'un contrôle des jeunes Daphnies par les Cyclopides adultes qui évitent ainsi une possible compétition trophique pour les stades copépodites herbivores.
Bosmina longirostris en revanche, supplante Daphnia tout au long de l'année. Cette espèce présente une première phase de croissance au début de l'été. Le 11 juillet, avec 14 ind./litre, le Bosminidae domine le peuplement (65,5\% des Crustacés).

En automne, quoique toujours prédominante, Bosmina développe de plus faibles effectifs (de 2 à 6 ind./l).

L'échantillonnage réalisé en 1985 donne une image du peuplement hivernal et printanier de la retenue (fig. 6a, 6b).

Toute cette période est dominée à plus de $80 \%$ par les Copépodes, essentiellement Diaptomides en hiver, relayés par les Cyclopides à la fin du printemps.

Les densités hivernales se situent au-dessous de 6 ind. /litre pour Diaptomus. Les forts effectifs de Cyclopides observés au mois de juin sont dus à de nombreux stades jeunes (49 ind.// le 3 juin dont $93 \%$ de stades naupliens).

Début juin voit l'essor de deux espèces de Cladocères avec une nette dominance de Bosmina longirostris.

En 1986, trois séquences se distinguent aisément dans le développement des Crustacés (fig. 7a, 7b) :

- une première phase de développement fin juin à mi-août dominée par les Cladocères et particulièrement par Daphnia qui représente en juinjuillet plus de $50 \%$ des Crustacés (densité maximale le 30 juin: 17,5 ind./);

- une deuxième phase caractérisée par le développement en 

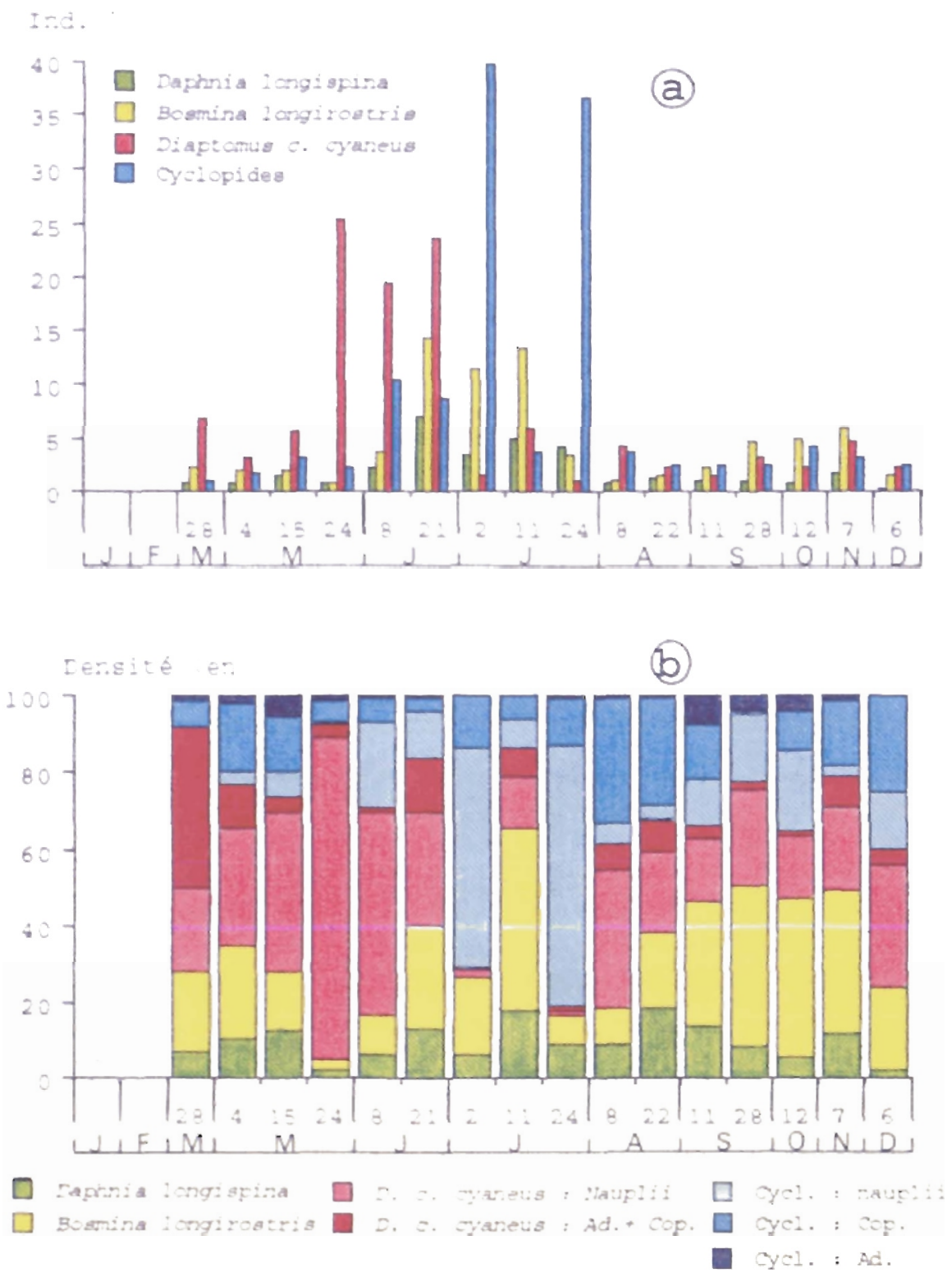

Fig. 5. - Structure du peuplement zooplanctonique en 1984 ; $a$ : densité, b: composition relative. Fig. 5. - Structure of the zooplankton population in 1984; a: density, b: relative abundance. 
Ind. $/ 1$

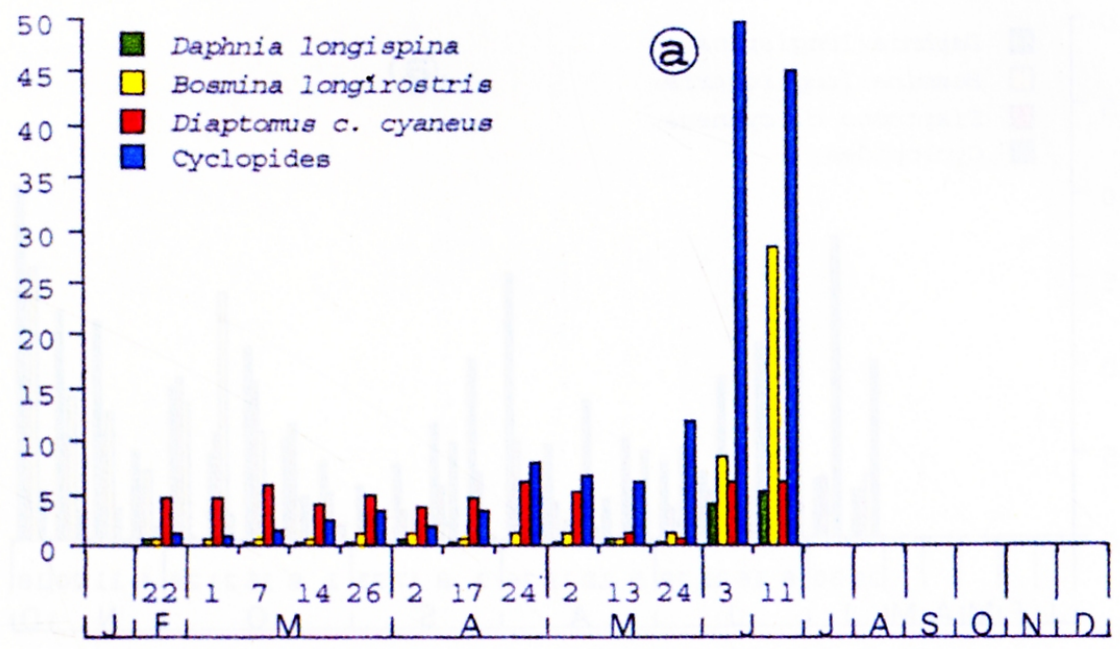

(b)

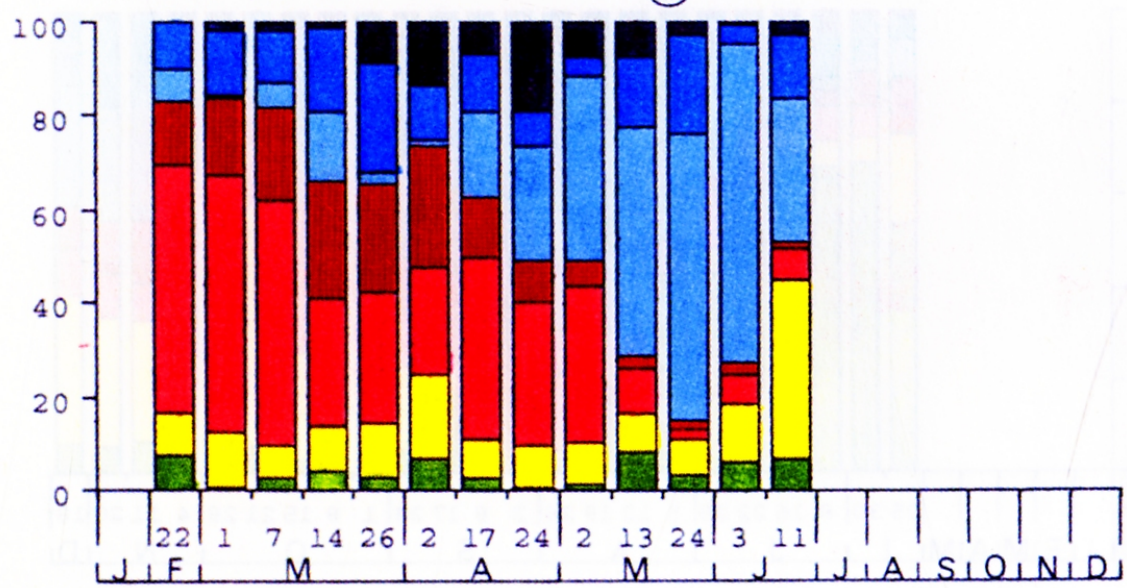

$\square$ Daphnia longispina $\square$ D. C. cyaneus : Nauplii $\square$ Cycl. : Nauplii
$\square$ Bosmina longirostris $\square$ D. c. Cyaneus : Ad.+ Cop. $\square$ Cycl. : Cop.
$\square$ Cycl. : Ad.

Fig. 6. - Structure du peuplement zooplanctonique en 1985; a : densité, b : composition relative. Fig. 6. - Structure of the zooplankton population in'1985; a: density, b: relative abundance. 
Ind. $/ 1$

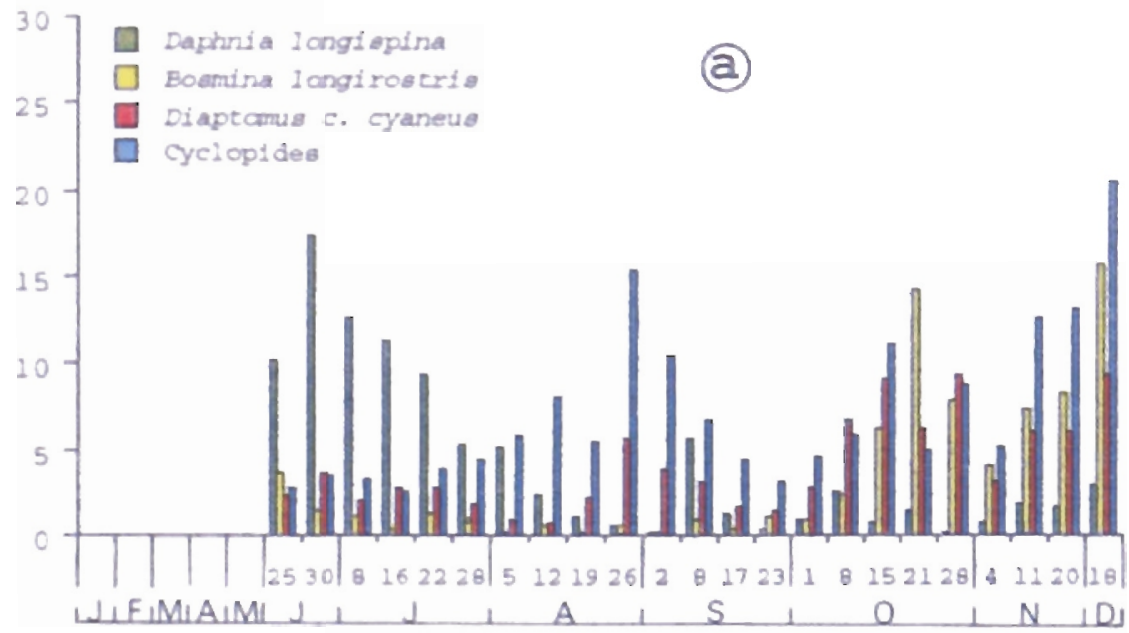

Densite (en is)

(b)
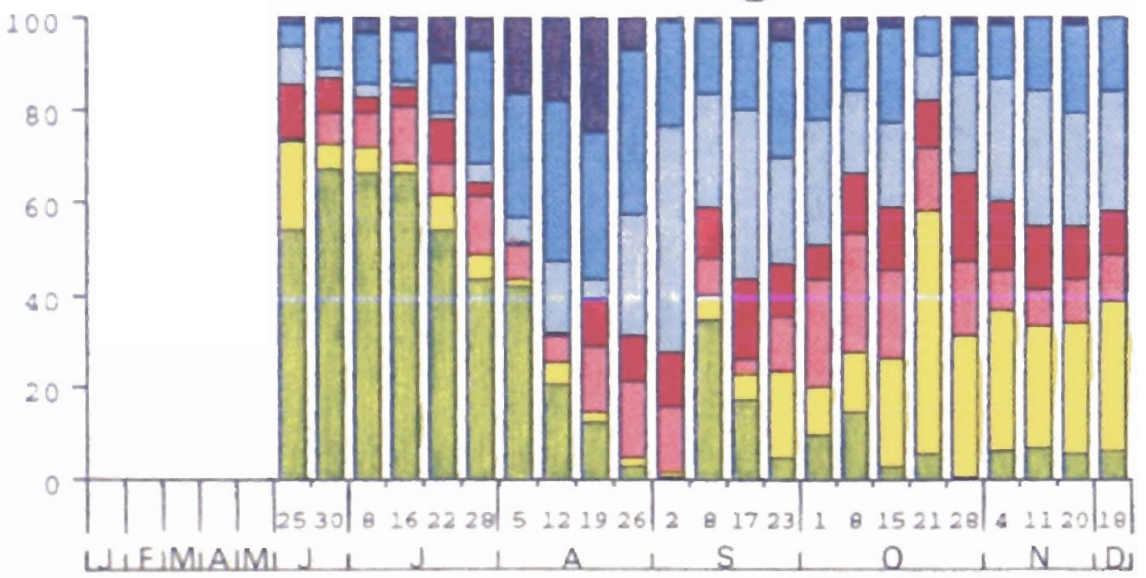

$\square$ Daphria Iongiepina

口 Boumina longiroetris

$\square$ D. C. Cyaneus : Maplit D. C. cyaneus : Ad. + Cop.

$\square$ Cyel. : Naupi!:

$\square$ Cyel. : Cop.

Q Cycl. : Ad.

Fig. 7. - Structure du peuplement zooplanctonique en 1986; a : densité, b: composition relative. Fig. 7. - Structure of the zooplankton population in 1986: a: density, b: relative abundance. 
août-septembre des Copépodes, majoritairement Cyclopidae;

- une phase automnale encore marquée par la dominance des Copépodes, mais montrant un important développement de la population de Bosmina qui atteint sa croissance maximale durant cette saison (15 et 17 ind./l les 21 octobre et 18 décembre respectivement).

Comme dans les observations précédentes, le peuplement hivernal et printanier en 1987, se caractérise par la domination des Copépodes (65 à $70 \%$ ), avec une légère suprématie des Diaptomides en mars-avril et des Cyclopides en mai (fig. 8a, 8b).

Durant cette période, on note, comparativement aux autres années, une contribution relativement plus importante des Cladocères et notamment du Bosminidae qui atteint son maximum le 10 juin (10 ind./I).

De juin à fin août, le zooplancton est dominé par des Cladocères (près de $70 \%$ des Crustacés), avec Daphnia comme représentant essentiel (environ $65 \%$ de la population estivale des Cladocères). Le déclin des Cladocères en août s'accompagne d'un nouvel essor des Copépodes en septembre.

La phase automnale de développement du zooplancton montre la composition habituellement observée dans le lac de Pareloup durant cette saison: prédominance de Bosmina longirostris en octobre-novembre (deux pics à 8 ind.// les 27 octobre et 4 novembre), progressivement sup- plantée par les Cyclopides en novembre-décembre.

\subsection{Répartition verticale du peuplement zooplanctonique}

La répartition verticale des trois groupes zooplanctoniques a été étudiée en 1986 et en 1987. Leur distribution dans la masse d'eau est figurée en période de stratification thermique et en période d'isothermie (fig. 9, 10, 11).

L'analyse des résultats montre que, en période de stratification thermique, la densité des Cladocères (fig. 9) diminue régulièrement avec la profondeur. Les Cladocères occupent toujours préférentiellement la couche épilimnique. L'hypolimnion reste peu peuplé bien que Baucheff-Nielsen (1988) signale, pour l'été 1984 une poche de forte densité en profondeur constituée de Daphnies ovigères et de néonates. Les pics observés en juillet 1986 et 1987 correspondent au développement estival de Daphnia longispina.

En période d'isothermie toute la masse d'eau est colonisée. Les maxima enregistrés durant cette période sont dus au développement automnal de Bosmina longirostris.

Globalement, en phase de stratification, les Copépodes (fig. 10) paraissent occuper également épilimnion et thermocline. Seul I'hypolimnion apparait moins peuplé. Cependant les observations plus approfondies effectuées en 1983, 1984 et 1985 (Baucheff-Nielsen, op. cit.), révèlent que 
Ind. /

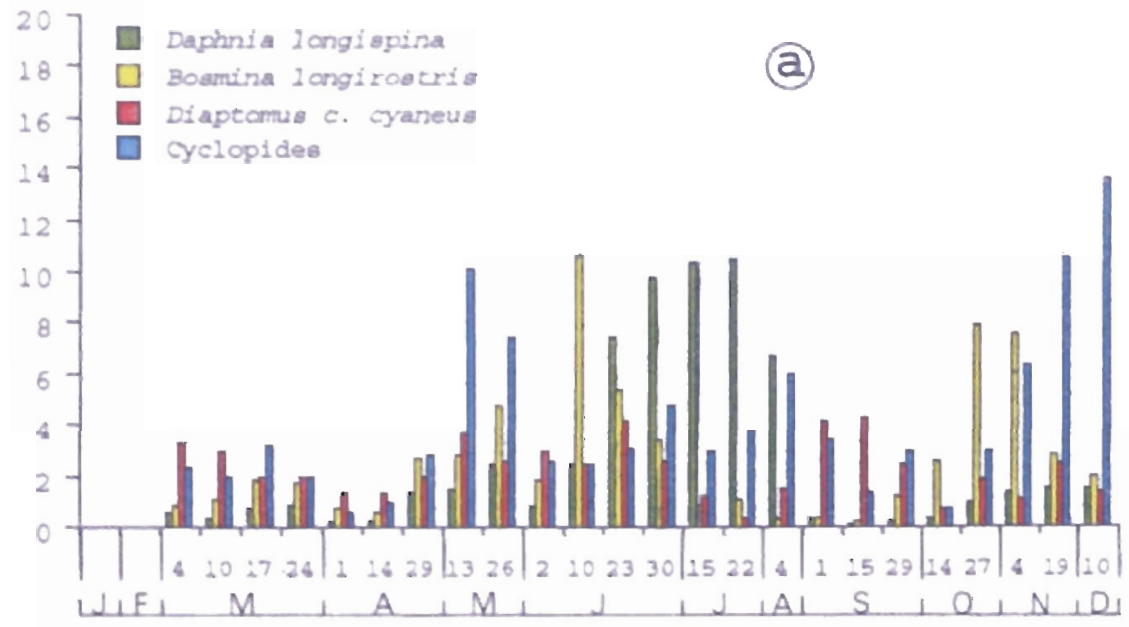

(b)

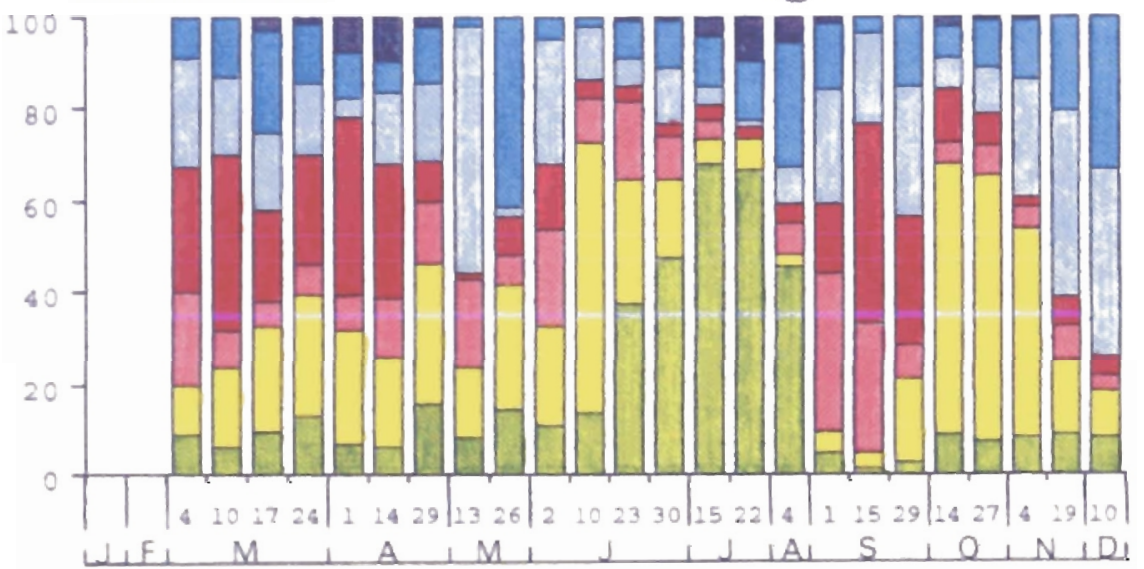

D Daphria langigpina $\square$ D. C. Cyaneus: Nauplit $\square$ Cycl. : Nauplit

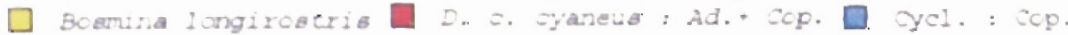

Q gral. : Ad.

Fig. 8. - Structure du peuplement zooplanctonique en 1987; a: densité, b: composition relative. Fig. 8. - Structure of the zooplankton population in 1987; a: density, b: relative abundance. 


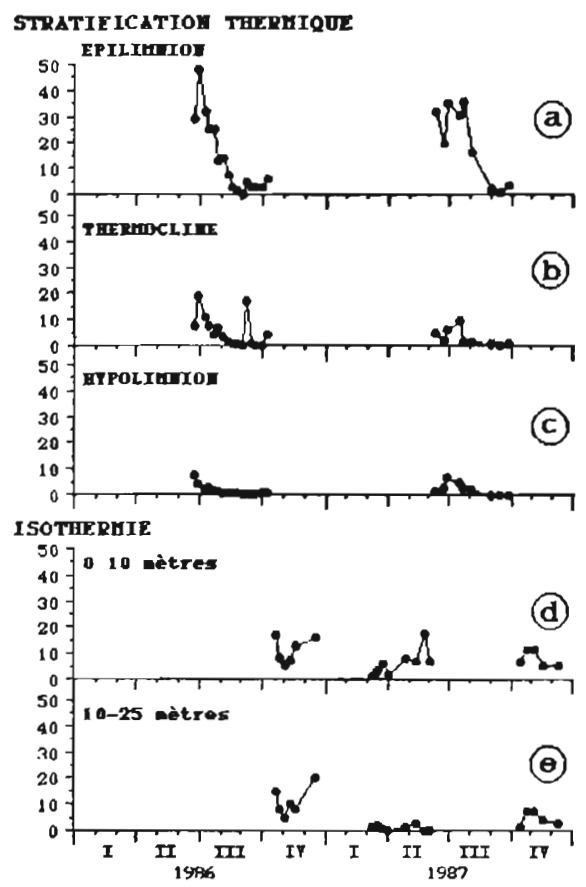

Fig. 9. - Répartition verticale des Cladocères en période de stratification thermique et d'isothermie (1986, 1987).

Fig. 9. - Vertical distribution of Cladocerans during thermal stratification and isothermia (1986, 1987).

Diaptomus cyaneus cyaneus colonise toute la colonne d'eau, quelle que soit la catégorie d'individus considérée. Les Cyclopides, par contre, se caractérisent par une concentration sur le fond de leurs stades naupliens. Ainsi, l'abondance maximale observée fin août-début septembre 1986 dans l'hypolimnion (fig. 10c) correspond aux pics de densité enregistrés aux mêmes dates et constitués d'une forte proportion de nauplii (fig. 7a, 7b).

En phase d'isothermie, l'occupation de la masse d'eau, quasi uniforme en 1986, montre, en 1987, une occupa-

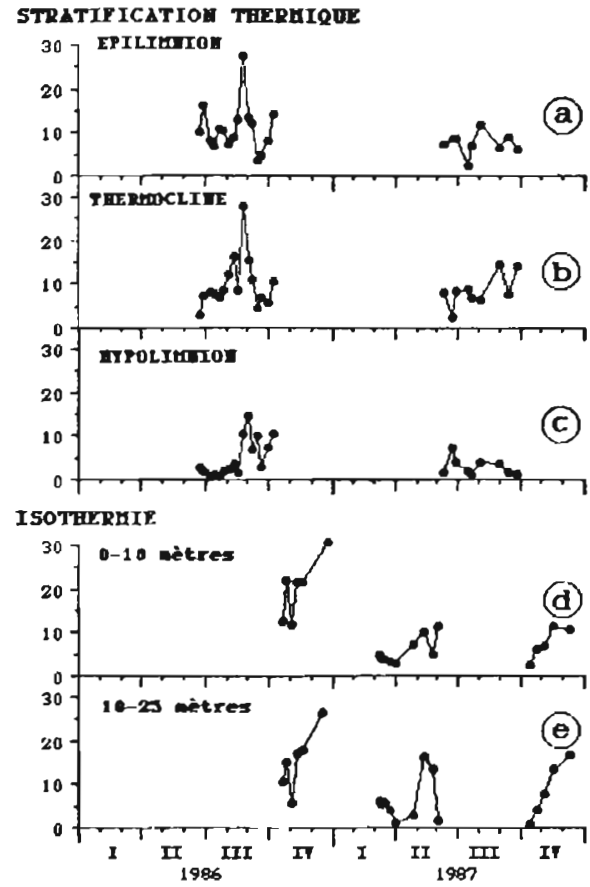

Fig. 10. - Répartition verticale des Copépodes en periode de stratification thermique et d'isothermie (1986, 1987).

Fig. 10. - Vertical distribution of Copepods during the thermal stratification and the isothermia period (1986, 1987).

tion préférentielle des couches profondes du lac. Comme précédemment, les maxima observés en profondeur au printemps et en automne 1987 (fig. 10e), coïncident avec les pics à forte proportion de nauplii enregistrés les 13 mai, 19 novembre et 10 décembre de la même année (fig. $8 a, 8 b$ ).

La densité des Rotifères augmente avec la profondeur en milieu stratifié (fig. 11).

En période d'isothermie, la masse d'eau est à peu près uniformément colonisée. Les densités sont, en 1986 , 
légèrement supérieures dans l'épilimnion (fig. 11d, 11e). En 1987, en revanche, la zone profonde est nettement plus peuplée.

Globalement, on observe que les Cladocères colonisent majoritairement la zone euphotique (couche épilimnique), tandis que les Copépodes occupent toute le colonne d'eau, avec une préférence des Cyclopides jeunes pour la zone profonde.

Contrairement aux Crustacés, les Rotifères paraissent peupler préférentiellement les couches profondes du lac.

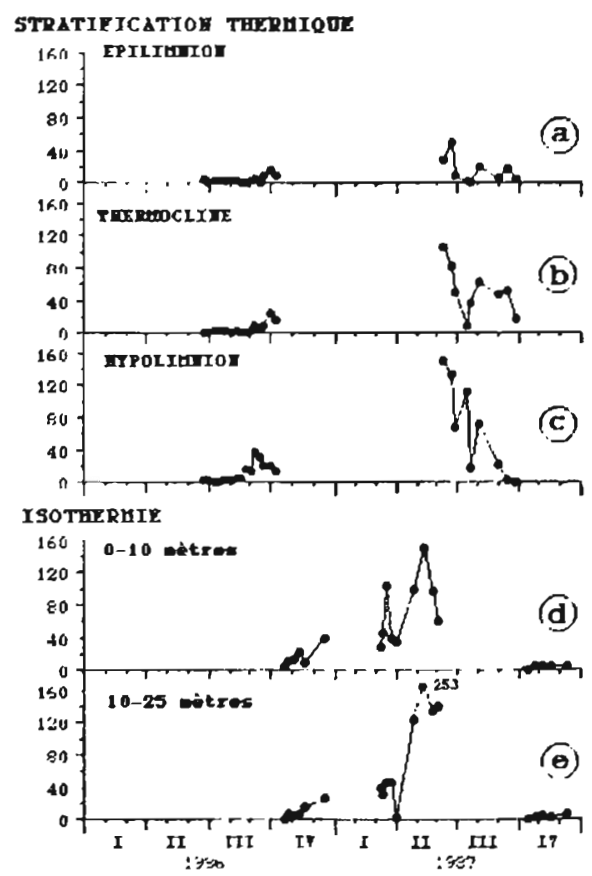

Fig. 11. - Répartition verticale des Rotifères en période de stratification thermique et d'isothermie (1986, 1987).

Fig. 11. - Vertical distribution of Rotifers during the thermal stratification and the isothermia period $(1986,1987)$.

\subsection{Impact des populations de Crustacés sur la communauté phytoplanctonique}

L'impact des populations de Crustacés sur la communauté phytoplanctonique peut être précisé par l'évolution saisonnière des proportions de chaque groupe de consommateurs (fig. 12a, 12b).

Sur un plan numérique, les petits brouteurs dominent le peuplement avec une moyenne de $56,6 \%$ sur les cinq années. En revanche, ils ne représentent que 10 à $20 \%$ de la biomasse hormis en automne 1986 et 1987 où ils atteignent près de $40 \%$.

Les grands brouteurs représentent en moyenne $40 \%$ de la biomasse et constituent le groupe le plus important.

Quoique peu nombreux les herbivores ont une biomasse supérieure à celle des petits brouteurs.

La compétition est évitée par l'alternance des périodes d'abondance des différents filtreurs, bien visible en 1986 et 1987 (fig. 12a, 12b).

Les camivores ont une amplitude variable. Dans le cadre des observations effectuées, les densités et biomasses maximales sont relevées au printemps (1984, 1985) et en été (1986, 1987).

L'impact du broutage dépend de la filtration potentielle de chaque brouteur. Les taux de filtration moyens individuels calculés pour les différents filtreurs par Baucheff-Nielsen (1988) ont été multipliés par la densité de ces groupes afin d'estimer la contribution 

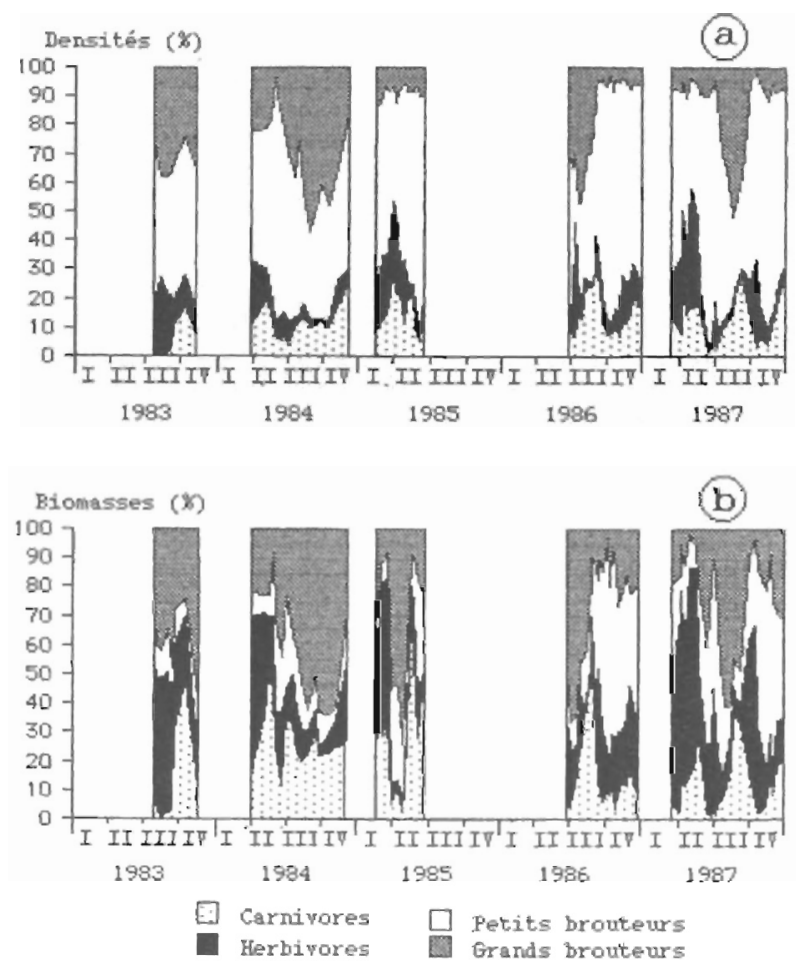

Fig. 12. - Composition reiative de la densité (a) et de la biomasse (b) des différentes catégories de consommateurs de 1983 à 1987.

Fig. 12. - Relative composition of the density (a) and biomass (b) of the different zooplanktongrazing classes, 1983 to 1987.

de chaque catégorie au taux de filtration potentiel.

La figure 13 rend compte de la pression de broutage dans la retenue au cours de la période d'étude.

A la fin de l'hiver et au début du printemps, l'impact des herbivores et des petits brouteurs est prépondérant. L'ensemble Diaptomidae-Bosminidae domine alors le peuplement crustacé.

Avec l'arrivée de l'été, l'influence de ces deux groupes diminue au profit des grands brouteurs qui deviennent les principaux régulateurs de la population algale.

Le Cohu et al. (1991) soulignent les faibles valeurs globales de la biomasse phytoplanctonique en période estivale malgré une forte production primaire (Capblancq et al., 1988).

En automne, l'augmentation de la densité des Bosminidae et des stades naupliens des Cyclopides, entraîne une contribution maximale des petits brouteurs. 


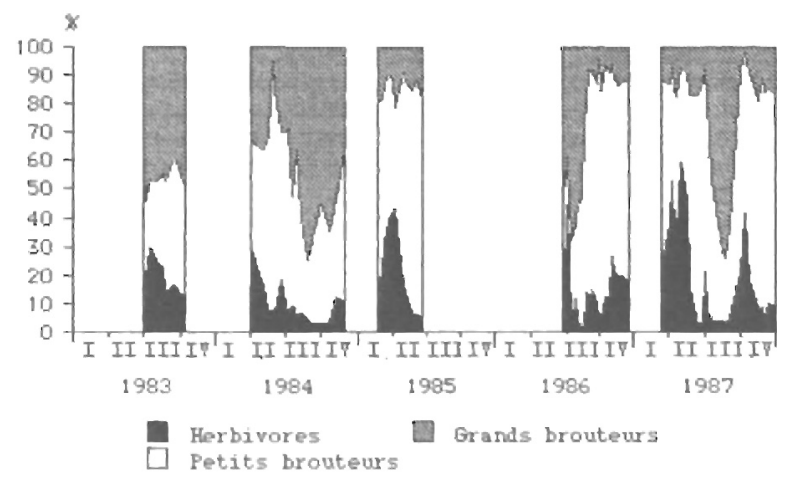

Fig. 13. - Contribution des trois catégories de brouteurs au taux de filtration potentiel de 1983 à 1987.

Fig. 13. - Contribution of the three filter-feeding classes to the potentiel filtering rate from 1983 to 1987 .

\section{DISCUSSION}

L'étude du peuplement zooplanctonique du lac de Pareloup effectuée de 1983 à 1987 révèle une grande variabilité interannuelle tant au niveau de la densité et de la biomasse des populations que de la structure du peuplement.

Si l'on compare le développement estival des populations de Crustacés (tab. I), on observe:

Tableau I. - Biomasses moyennes (B: $\mu \mathrm{g}$ P.S./I) des composantes zooplanctoniques et contribution relative (\%) des différents groupes de Crustacés en période estivale (juinjuillet-août; 1983 : juillet-août).

Table I. - Average biomass (B: $\mu$ g D.W./l) of zooplankton, and relative contribution (\%) of the different crustacean groups during summer (June-July-August; 1983: July-August).

\begin{tabular}{|l|r|r|r|r|r|r|r|r|}
\hline \multicolumn{1}{|c|}{ Taxons } & \multicolumn{3}{|c|}{1983} & \multicolumn{2}{c|}{1984} & \multicolumn{2}{|c|}{1986} & \multicolumn{2}{|c|}{1987} \\
\hline & B & $\%$ & B & $\%$ & B & $\%$ & B & $\%$ \\
\hline Daphnia longispina & 15,5 & 21 & 9,6 & 16 & 21,5 & 47 & 16,5 & 31 \\
\hline Bosmina longirostris & 12,0 & 17 & 18,1 & 31 & 1,8 & 4 & 24,3 & 46 \\
Cladocères & 27,5 & 36 & 27,8 & 47 & 23,3 & 51 & 40,8 & 77 \\
Diaptomus C. cyaneus & 42,3 & 58 & 11,6 & 20 & 7,9 & 17 & 4,8 & 9 \\
Cyclopides & 2,4 & 4 & 19,4 & 33 & 14,9 & 32 & 7,1 & 14 \\
Copepodes & 44,7 & 62 & 30,9 & 53 & 22,8 & 49 & 11,9 & 23 \\
Total crustacés & 72,2 & & 58,7 & & 46,1 & & 52,8 & \\
\hline Total zooplancton & 75,0 & & 60,9 & & 46,5 & & 65,4 & \\
Rotiferes & 2,8 & & 2,2 & & 0,4 & & 12,7 & \\
\hline
\end{tabular}


- la prédominance des Copépodes en 1983 (62\% de la biomasse moyenne des Crustacés), celle des Cladocères en 1987 (77\% de la biomase crustacéenne). En 1984 et 1986, le dévelopement des deux groupes est à peu près équilibré.

- le peuplement estival des Copépodes est généralement marqué par le développement des Cyclopides. En 1983 cependant, ce sont les Diaptomides qui, à eux seuls, constituent près de $90 \%$ du peuplement des Copépodes.

- la biomasse moyenne de l'ensemble des Crustacés varie de $72,2 \mu \mathrm{g}$ P.S./I en 1983 à $46,1 \mu \mathrm{g}$ P.S./I en 1986.

- la biomasse copépodienne est maximale en 1983 (44,7 $\mu \mathrm{g}$ P.S.I, $62 \%$ de la biomasse des Crustacés). Elle est due au grand développement de Diaptomus c. cyaneus. En 1987, année où les deux groupes de Copépodes montrent leur plus faible développement, elle est réduite d'un facteur 4.

- la biomasse cladocérienne, maximale en 1987 (40,8 $\mu \mathrm{g}$ P.S./I) est presque 2 fois moindre en 1986.

- sauf en 1983, année où la contribution des deux Cladocères est à peu près identique, on observe la prédominance d'une espèce sur l'autre: prédominance de Bosmina longirostris en 1984 et 1987 (respectivement 65 et $60 \%$ de la biomasse cladocérienne), prédominance de Daphnia longispina en 1986 (92\% de cette biomasse) alors qu'en 1984, cette même espèce montre sa plus faible contribution $(16 \%$ de la biomasse totale des Crustacés).

L'évolution saisonnière du zooplancton dépend des facteurs abiotiques (température, lumière) et de facteurs biotiques (disponibilités nutritives, compétition, prédation) qui caractérisent le milieu.

Dans le lac de Pareloup, il semble que la pression de prédation exercée par les poissons zooplanctophages, plus ou moins intense selon le recrutement, puisse expliquer pour une grande part les variations interannuelles enregistrées.

Dans la retenue, les deux espèces dominantes sont le Gardon (Rutilus rutilus) et la Perche (Perca fluviatilis). La période de frai se situe d'avril à juin. Des variations de niveau du plan d'eau durant cette période peuvent, en découvrant les frayères, limiter fortement les éclosions, donc le recrutement, et, ainsi, diminuer considérablement la prédation exercée par les alevins.

Les alevins de perche et de gardon exercent une prédation sélective du printemps au début de l'été. Les adultes de gardon continuent à exploiter le zooplancton en période estivale (Richeux et al., 1992).

La préférence alimentaire des gardons pour les Cladocères est soulignée par plusieurs auteurs. Hammer (1985) note que les Copépodes sont rarement ingérés. Townsend et Perrow (1989) décrivent l'importance des Cladocères dans l'alimentation des jeunes gardons. 
Etudiant les indices alimentaires, Reyes-Marchand (1993) place les Cladocères comme proies essentielles.

Pour le lac de Pareloup, les travaux effectués sur les poissons zooplanctophages de la retenue (analyse des contenus stomacaux, expérimentation en enceinte) par Richeux et al. (1992, 1994), Dubois (1991), Dubois et al. (1994), mettent en évidence une prédation sélective des Cladocères par le Gardon, des Copépodes par la Perche.

Des changements dans la dynamique de population de ces deux prédateurs auront des répercussions (en particulier, une prédation moindre ou plus intense) sur le développement des populations-proies, entraînant ainsi la prédominance de l'un ou l'autre de ces groupes zooplanctoniques comme cela a été observé en 1983 et 1987.

La prédominance de Daphnia ou de Bosmina au sein de la communauté pélagique est vraisemblablement liée au stock des poissons zooplanctophages.

Hrbácek (1962), Brooks et Dodson (1965), observent une diminution de la taille moyenne du zooplancton sous l'effet de la prédation. Kitchell et Carpenter (1988), dans une étude expérimentale sur la structure du réseau trophique planctonique dans le lac Tuesday, montrent, concernant le compartiment zooplancton, que l'augmentation ou la diminution du nombre des poissons zooplanctophages dans la communauté pélagique entraîne un changement de la composition du zooplancton filtreur.

En présence d'un stock important de prédateurs (Vairons, dans le lac Tuesday), le zooplancton est composé d'une majorité de formes de petite taille (Bosmina longirostris, Rotifères, Cyclopides). L'élimination ou la diminution des prédateurs se traduit par une dominance des grandes espèces (Daphnia pulex, Holopedium gibberum).

Selon ce schéma, la composition du zooplancton observée en 1984 et 1987 : dominance à plus de $65 \%$ des formes de petite taille (B. longirostris, Cyclopides, Rotifères) témoigne d'une forte prédation des poissons zooplanctophages et d'effectifs conséquents de prédateurs.

En 1986 en revanche, le recrutement semble avoir été limité, réduisant la pression de prédation et favorisant de ce fait le développement de $D$. longispina observé cette même année (tab. I, fig. 7a, 7b).

Les fortes densités de zooplancton (90 ind./I selon les premières estimations numériques) et l'abondance des formes de grande taille (Daphnia essentiellement) observées à Pareloup un an après la vidange du lac (juin 1993), paraissent confirmer le rôle essentiel joue par les poissons zooplanctophages dans le développement des populations et la structure de la communauté zooplanctonique de la retenue.

Les études actuellement menées (relations zooplancton-bactérioplancton, valeur nutritive des planctontes, 
impact des poissons planctophages) devraient permettre une approche plus précise des interactions existantes entre le compartiment zooplancton et les niveaux adjacents du réseau trophique.

\section{RÉFÉRENCES}

Baucheff-Nielsen P., 1988. Contribution à l'écologie du zooplancton du lac de Pareloup. Thèse $3^{\text {eme }}$ cycle. U.P.S., Toulouse: $104 \mathrm{pp}$.

Bottrell H.H., Duncan A., Gliwics Z.W., Grygierek E., Herzig A., Hillbricht-llkowska A., Kurusawa H., Larsson P. \& Weglenska J., 1976. Contribution from the plankton ecology group. A review of some problems in zooplankton production studies. Nonw. J. Zool., 24 : 419-456.

Brooks J. \& Dobson I., 1965. Predation, body size and composition of plankton. Science, $150: 28-35$.

Capblancq J., Thebault J.M. \& Jrad A., 1988. Relations entre la lumière et la photosynthèse du phytoplancton dans un réservoir mésotrophe (Pareloup): variations saisonnères des paramètre. Ann/s. Limnol., 24 : 39-48.

Dubois C., 1991. Utilisation de résultats d'expériences menées en enceintes (été 1988 au lac de Pareloup (Aveyron)) : stratégies alimentaires des alevins de perches (Perca fluviatilis L.) et de gardon (Rutilus rutilus L.) vis à vis du zooplancton. D.E.A, U.P.S, Toulouse: $39 \mathrm{pp}$.

Dubois C., Richeux C., Tourenq J.N. \& Le Jolivet C., 1994. Régime et comportement alimentaire du gardon (Rutilus rutilus L.) et de la perche (Perca fluviatilis L.) de la retenue de Pareloup.
I. Les alevins. Hydroécol. Appl. 6 (1/2) : pp. 227-242.

Dumont H.S., Vandevelde I. \& Dumont S., 1975. The dry weight estimate of biomass in a selection of Cladocera, Copepoda and Rotifera from plankton, periphyton and benthos of continental waters. Oecologia, 19 : 75-97.

Hammer C., 1985. Feeding behaviour of roach (Rutilus rutilus L.) larvae and the fry of perch (Perca fluviatilis L.) in lake Lankau. Arch. Hydrobiol., 103 (1) : 61 74.

Hrbácek J., 1962. Species composition and the amount of zooplankton in relation to the fish stock. Pozpr. Ceskol. Akad. Ved., $72:$ 1-116.

Kitchell J.F. \& Carpenter S.R., 1988. Foodweb manipulation in experimental lakes. Verh. Internat. Verein. Limnol., 23 : 351-358.

Labroue L., 1990. Echanges sédimentseau dans le lac de Pareloup. Colloque Hydroécologie EDF Paris : 6-7.

Lavandier P. 1990. Dynamics of bacterioplankton in a mesotrophic French reservoir (Pareloup). Hydrobiologia., 207 : 79-86.

Le Cohu R., Comoy N., Guitard J. \& Brabet J., 1991. Périodicité du phytoplancton dans un réservoir de moyenne profondeur: le lac de Pareloup (Massif Central, France), un exemple de succession cyclique. Annls. Limnol., 27(3) : 197-214.

Le Jolivet (C.) 1988. Contribution à l'étude du recrutement des poissons du réservoir de Pareloup (Aveyron). Thèse de Doctorat, I.N.P. Toulouse: $288 \mathrm{pp}$.

McQueen D.J., Post J.R. \& Mills E.L., 1986. Trophic relationships in freshwater pelagic ecosystems. Can. J. Fish. Aquat. Sci., 43: 1571-1581.

Reyes-Marchand P., 1993. Impact des alevins de gardons (Rutilus rutilus L.) sur les communautés planctoniques littorales. Modélisation du fonctionnement trophique du lac d'Aydat. Thèse de Doctorat, Université Blaise Pascal, La- 
boratoire de Zoologie, Clermont II. : $168 \mathrm{pp}$.

Richeux C., Arias-Gonzales J.E. \& Tourenq J.N. 1992. Etude du régime alimentaire des gardons (Rutilus rutilus L.) du lac de Pareloup (Massif Central, France). Annls. Limnol., 28 (3) : 245252.

Richeux C., Nogues J.F., Tourenq J.N. \& Aragon B., 1994. Inventaire piscicole de la retenue hydroélectrique de $\mathrm{Pa}$ reloup lors de la vidange de juin 1993. Essai d'un nouveau système d'acquisition et de traitement des signaux d'un échosondeur. Hydroécol. Appl. 6 (1/2) : 197-226.

Santer B., 1993. Do Cyclopoid control Daphnia populations in early spring, the- reby protecting their juvenile instar stages from food limitation? Verh. Internat. Verein. Limnol., 25 : 634-637.

Spruies W.G. 1984. Towards an optimal classification of zooplankton for lake ecosystem studies. Verh. internat. Verein. Limnol., 22 : 320-325.

Thébault J.M. \& Salençon M.J. 1993. Simulation model of a mesotrophic reservoir (Lac de Pareloup, France): Biological model. Ecol. Modelling, 65 : 1-30.

Townsend C.R. \& Perrow M.R., 1989. Eutrophication may produce population cycles in roach (Rutilus rutilus L.), by two contrasting mechanisms. J. Fish. Biol., 34 : 161-164. 